\title{
ReSEARChArTicle
}

\section{Weed management strategies in Bt cotton under Humid Southern Plain Zone of Rajasthan}

\author{
HARPHOOL MEENA, P.K.P. MEENA AND B.L. KUMHAR
}

\section{SUMMARY}

A field experiment was conducted during two consecutive years of Kharif 2012 and 2013 at Agricultural Research Station, Banswara to find out suitable weed management strategies for Bt cotton. The experiment was laid-out in Randomized Block Design with three replications having nine treatments. Results revealed that, the application of pendimethalin $30 \%$ EC @ $0.75 \mathrm{~kg}$ a.i. / ha PE $f b$ quizalofop-P- ethyl $50 \mathrm{~g}$ a.i. / ha at 20-30 DAS + one hoeing gave significantly higher bolls plant $^{-1}(36.30)$, boll weight $(4.22 \mathrm{~g})$, seed cotton yield $\left(2275 \mathrm{~kg} \mathrm{ha}^{-1}\right)$, net return (Rs.55581/- ha $\left.{ }^{-1}\right)$ and B:C (2.70) over rest of treatments, but it was found at par with weed free check and application of pyrithiobac sodium @ $62.5 \mathrm{~g}$ a.i./ ha $f b$ quizalofop-P- ethyl $50 \mathrm{~g}$ a.i./ha at 20-30 DAS + one hoeing bolls plant ${ }^{-1}(34.84)$, boll weight (4.19 g), seed cotton yield (2251 $\mathrm{kg} \mathrm{ha}^{-1}$ ), net return (Rs.54669/- ha $\mathrm{f}^{-1}$ ) and B:C (2.64). The maximum weed control efficiency (60.75\%), lowest weed population $\left(12.39 \mathrm{~m}^{-2}\right)$ and weed dry matter accumulation $\left(14.63 \mathrm{~g} \mathrm{~m}^{-2}\right)$ at $60 \mathrm{DAS}$ were observed under application of pendimethalin 30\% EC @ $0.75 \mathrm{~kg}$ a.i. / ha PE $f b$ quizalofop-P- ethyl $50 \mathrm{~g}$ a.i. / ha at 20-30 DAS + one hoeing over rest of treatments. However, it was found at par with weed free check and application of pyrithiobac sodium @ 62.5 g a.i./ ha $\mathrm{fb}$ quizalofopP- ethyl $50 \mathrm{~g}$ a.i./ha at 20-30 DAS + one hoeing.

Key Words : Cotton, Pendimathlin, Pyrithiobac sodium, Weed control efficiency

How to cite this article : Meena, Harphool, Meena, P.K.P. and Kumhar, B.L. (2017). Weed management strategies in Bt cotton under Humid Southern Plain Zone of Rajasthan. Internat. J. Plant Sci., 12 (2): 243-248, DOI: 10.15740/HAS/IJPS/12.2/ 243-248.

Article chronicle : Received : 01.05.2017; Revised : 01.06.2017; Accepted : 18.06.2017

\section{MEMBERS OF THE RESEARCH FORUM}

Author to be contacted :

HARPHOOL MEENA, AICRP on Irrigation Water Management, Agricultural Research Station, Agriculture University, KOTA (RAJASTHAN) INDIA

Email : hpagron@rediffmail.com

Address of the Co-authors:

P.K.P. MEENA AND B.L. KUMHAR, AICRP on Irrigation Water Management, Agricultural Research Station, Agriculture University, KOTA (RAJASTHAN) INDIA 